\title{
Pour une approche communicationnelle des organisations de mouvement social
}

\section{Florence Carion}

\section{(2) OpenEdition \\ 12 Journals}

Édition électronique

URL : http://journals.openedition.org/communicationorganisation/124

DOI : 10.4000/communicationorganisation. 124

ISSN : $1775-3546$

Éditeur

Presses universitaires de Bordeaux

Édition imprimée

Date de publication : 1 juillet 2007

Pagination : 64-77

ISSN : 1168-5549

\section{Référence électronique}

Florence Carion, « Pour une approche communicationnelle des organisations de mouvement social », Communication et organisation [En ligne], 31 | 2007, mis en ligne le 01 juillet 2010, consulté le 19 avril 2019. URL : http://journals.openedition.org/communicationorganisation/124 ; DOI : 10.4000/ communicationorganisation. 124 
Dossier : Migrations conceptuelles. D'où viennent les concepts de la communication organisationnelle?

\title{
Résumé
}

Dans le cadre de notre recherche, nous souhaitons étudier les organisations du mouvement social altermondialiste, avec un regard communicationnel. Cette recherche s'inscrit dans la lignée des travaux en communication organisationnelle qui s'intéressent aux phénomènes de constructions et de transformations organisationnelles. Les organisations n'y sont plus considérées comme des données au sein desquelles il est possible d'étudier les phénomènes ou politiques de communication; ils traitent davantage du rôle constitutif de la communication dans les phases de construction de l'organisation. Le présent article propose de retracer le parcours qui nous a amenée à constituer ce cadre théorique et méthodologique de recherche mêlant approches sociologiques et communicationnelles, qui nous ouvre des pistes concrètes d'analyse de la construction identitaire des organisations, à travers l'évolution de leurs discours.

Mots clés : mouvement social, organisations altermondialistes, construction identitaire des organisations.

\begin{abstract}
This research analyses the "alter-globalization organisations" with a communicational approach. Organisations are not only places where communicational phenomenon and policies can be studied: communications plays also some functions in the building of the organisation. This article focuses on the theorical and methodological path we have followed to elaborate our scientific frame on work. It opens ways to improve the understanding of organisational identities through their discourses.
\end{abstract}

Keywords: alter-globalization organisations, social movement, organisational identities, organisational discourses.

Florence Carion est doctorante au Laboratoire d'Analyse des Systèmes de Communication des Organisations (LASCO) de l'Université Catholique de Louvain. Ses travaux de recherche visent à élaborer un cadre d'analyse sociologique et médiologique pour l'étude de la genèse et du parcours des organisations de mouvements sociaux, en particulier les organisations altermondialistes. 


\section{Pour une approche communicationnelle des organisations de mouvement social Florence Carion}

carion@reco.ucl.ac.be

Dans le cadre de notre recherche, nous souhaitons étudier les organisations du mouvement social altermondialiste, avec un regard communicationnel. Ce mouvement social, encore récent, fait de plus en plus l'objet de travaux scientifiques. Une porte d'entrée à l'analyse de la nouvelle forme de rassemblement et de cohésion que constitue ce mouvement peut être celle du phénomène organisationnel qui s'y révèle. Cette recherche s'inscrit dans la lignée des travaux en communication organisationnelle qui s'intéressent aux phénomènes de constructions et de transformations organisationnelles ${ }^{1}$. Les organisations n'y sont plus considérées comme des données au sein desquelles il est possible d'étudier les phénomènes ou politiques de communication; ils traitent davantage du rôle constitutif de la communication dans les phases de construction de l'organisation.

Dès le départ, et ce afin de comprendre les spécificités des organisations de mouvement social, notre recherche s'est nourrie des apports de la sociologie des organisations, des mouvements sociaux et des associations. En croisant les apports de ces travaux sociologiques et des théories de la communication, on constate qu'ils s'articulent et se complètent, permettant ainsi de définir une approche communicationnelle des organisations de mouvement social. Les organisations y sont pensées en tant que systèmes de communication; la communication est un élément structurant les organisations, elle est organisante. Cette approche nous amène à nous intéresser plus particulièrement à la dimension identitaire des organisations, à travers leurs discours. L'analyse de ces « textes » accompagnant les processus de constitution et de transformation organisationnelles que nous proposons repose à nouveau sur une articulation, entre les méthodologies utilisées en sociologie des mouvements sociaux (l'analyse des cadres) et en information et communication (l'analyse sémio-pragmatique).

\footnotetext{
${ }^{1}$ Ces travaux, dépassant l'étude de la communication instrumentale des organisations, s'attachent à analyser les phénomènes de communication contribuant au fait organisationnel. Voir à ce propos Brulois V., «Communication et organisation : état des recherches en sciences de l'information et de la communication ", in Education permanente, $\mathrm{n}^{\circ} 16,2006$, pp. 29-41.
} 
Dossier : Migrations conceptuelles. D'où viennent les concepts de la communication organisationnelle?

Le présent article propose de retracer le parcours qui nous a amenée à constituer ce cadre théorique et méthodologique de recherche mêlant approches sociologiques et communicationnelles, qui nous ouvre des pistes concrètes d'analyse de la construction identitaire des organisations, à travers l'évolution de leurs discours.

\section{Un cadre d'analyse sociologique}

Le fil conducteur de notre recherche étant le rôle constitutif des discours dans la formation ou la transformation de l'organisation de mouvement social, nous nous devions de nous tourner vers la sociologie des organisations, des mouvements sociaux et des associations, pour pouvoir comprendre les liens entre les discours, les structures, les fonctionnements et les interactions en organisations. Nous présentons ci-après les principaux apports des approches sociologiques mobilisées.

\section{La question organisationnelle dans l'analyse des mouvements sociaux}

Les approches sociologiques nous ont avant tout permis de définir ${ }^{2}$ le mouvement social comme une nébuleuse rassemblant à la fois des individus isolés, des groupes, des organisations formalisées, constituant autant de niveaux de régulation de l'action collective. On constate que l'action collective est le moteur de cette conception des mouvements sociaux: ce sont des situations dans lesquelles se manifestent des convergences entre une pluralité d'agents sociaux, qui se réunissent avec une intention de coopération et agissent de manière concertée et revendicative.

Les travaux d'Erhard Friedberg ${ }^{3}$ et d'Erik Neveu ${ }^{4}$ nous ont invitée à dépasser la fausse dichotomie entre organisation et action collective, entre organisation et action organisée. Ces auteurs estiment qu'il est erroné de sous-estimer le degré d'organisation et de structuration des mouvements sociaux, et de surestimer la rigueur et l'originalité de la formalisation des rôles et des structures dans les organisations. E.

\footnotetext{
${ }^{2}$ Grâce aux travaux des sociologues Stefaan Walgrave, Donatella Della Porta et Mario Diani. Voir à ce propos Walgrave S., « Nieuwe sociale bewegingen : een type van sociale bewegingen », in : Tijdschrift voor Sociologie, 13, 1, pp. 71-104 et Della Porta D., Diani M., Social Movements. An Introduction, Oxford, Blackwell, 1999, p. 16.

${ }^{3}$ Friedberg E., «Les quatre dimensions de l'action organisée », in : Revue française de sociologie, vol. 33, 1992, 4, pp. 531-557.

${ }^{4}$ Neveu E., Sociologie des mouvements sociaux, Paris, La Découverte, Repères 207, 2000 .
} 
Friedberg conçoit l'organisation comme un niveau de régulation de l'action organisée. Il n'y a pas, selon lui, de différence de nature entre une organisation formalisée et des formes plus diffuses d'action collective, mais plutôt une différence de «degré ». Ainsi, l'auteur nous amène à penser qu'il existe un continuum entre l'action d'un individu, d'un groupe ou d'une organisation; il s'agit de différents niveaux de régulation.

D'autres approches sociologiques nous indiquent qu'il y a une pertinence importante à envisager la question organisationnelle au sein des mouvements sociaux. Selon E. Neveu, tout mouvement social qui tente de s'inscrire dans la durée pour atteindre ses objectifs est confronté à cette question ${ }^{5}$. Lilian Mathieu ${ }^{6}$ pointe également l'importance, pour les militants, de construire et de consolider la dimension collective de leur mobilisation, en se dotant d'une forme minimale d'organisation -ce qui renvoie à l'idée de degrés divers de l'action organisée- à même de maximiser le potentiel protestataire du groupe et d'assurer son existence dans la durée.

$\mathrm{Au}$ sein du mouvement social, les individus se rassemblent ou non, régulent leurs interactions à différents niveaux. Diverses formes sociales dynamiques apparaissent ainsi, se composent, se décomposent. Différents niveaux de "connexité » font se rassembler et s'organiser les individus.

\section{Les traits particuliers des organisations de mouvement social}

Les travaux relevant de la sociologie des mouvements sociaux et des associations $^{7}$ nous ont permis de déceler les particularités de ces organisations. Elles se caractérisent principalement par quatre éléments.

\footnotetext{
5 A ce propos, une étude intéressante réalisée par William Gamson est citée par l'auteur (p. 25) : ce chercheur, ayant défini des normes de «bureaucratisation » des mobilisations sociales aux Etats-Unis de 1800 à 1945 (statuts écrits, fichier d'adhérents, organisations à plusieurs échelons hiérarchiques), a conclu que la grande majorité des organisations répondant à ces critères parviennent à faire entendre leurs revendications à leurs interlocuteurs.

${ }^{6}$ Mathieu L., Comment lutter? Sociologie et mouvements sociaux, Paris, Les Editions Textuel, 2004.

${ }^{7}$ Principalement les travaux de D. Della Porta et M. Diani, H. Kriesi, J.-L. Laville et R. Sainsaulieu. Voir à ce propos Della Porta D., Diani M., Op. cit., Kriesi H., «Sviluppo organizzativo dei nuovi movimenti sociali e contesto politico», in: Rivista italiana di scienza politica, vol. 23, 1, 1993, cité dans Neveu E., Op. cit., et Laville J.-L., Sainsaulieu R., Sociologie de l'association, Paris, Desclée de Brouwer, 1997.
} 
Dossier : Migrations conceptuelles. D'où viennent les concepts de la communication organisationnelle?

Tout d'abord, ce sont des organisations fonctionnant en réseaux. D'une part, il existe des réseaux du point de vue des relations internes et interpersonnelles (différents circuits de communication sont présents au sein de l'organisation et les membres croisent les appartenances à d'autres associations ou groupes), et, d'autre part, se développent des réseaux interorganisationnels.

Ensuite, elles présentent une orientation d'action protestataire tournée vers l'extérieur. Ce sont des «groupes intégrés », pour reprendre les termes d'Anthony Oberschall ${ }^{8}$, entretenant des relations stables avec les autorités et ayant des relais vers l'extérieur. Afin de porter leurs revendications vers l'extérieur, les organisations de mouvement social sont amenées à développer des stratégies d'actions dont les répertoires se modifient avec le temps (par exemple, aux actions classiques grèves, pétitions- s'ajoutent depuis quelques dizaines d'années des actions " publicitaires », liées aux images de marque ; plus récemment encore est apparu le recours aux nouvelles technologies et à l'expertise).

Puis, elles favorisent la participation directe et solidaire des adhérents, selon des principes démocratiques. Les adhérents sont donc plutôt des membres actifs, qui peuvent être bénéficiaires potentiels de l'action, ou militants moraux. Leur engagement est solidaire et participatif dans la réalisation de l'œuvre collective. Il y a donc peu d'échelons hiérarchiques au sein de ce type d'organisations. La démocratie directe est favorisée, sous l'œil de leaders charismatiques ou experts.

Enfin, il faut, pour comprendre ces valeurs fédératrices permettant l'action collective et la participation des membres, caractériser les organisations de mouvement social par un niveau minimal de croyance : leurs buts de missions, ou leur projet associatif. Le projet est à l'origine du rassemblement des acteurs mais il explicite également le contrat d'adhésion de ceux-ci. Il est à la fois la base, la référence au bien commun partagé par les membres et un garde-fou en cas de désaccords ou de discussions. L'action collective, souvent protestataire, découle de cette mission commune. En effet, sur base du projet -et tout en continuant à le préciser-, les membres s'accordent pour se donner les moyens d'une action collective, ils s'entendent sur les principes de justification de leur projet et de leur action. Il s'agit là de la dimension institutionnelle de l'association, qui nous intéresse particulièrement.

${ }^{8}$ Oberschall A., Social conflict and social movements, Englewood Cliffs, Prentice Hall, 1973. 
$\mathrm{Si}$ ces quelques éléments peuvent permettre de caractériser les organisations de mouvement social et de les étudier précisément, il faut également envisager ce qu'Henry Mintzberg ${ }^{9}$ appelle le «cycle de vie » des organisations : la formation (ou la conquête selon Albert Meister ${ }^{10}$ ), le développement (ou la consolidation), la maturité et le déclin. Différentes configurations ou logiques, divers mondes sociaux pourraient se déceler grâce à cette analyse diachronique.

Comme nous l'avons vu, la sociologie des organisations nous invite à concevoir l'organisation comme une forme sociale dynamique, un niveau de régulation de l'action collective. L'acteur collectif d'une mobilisation est amené à cadrer son action, évoluant le long d'un continuum dans le degré de son organisation. La sociologie des mouvements sociaux et des associations nous permet d'aborder les traits particuliers des organisations de mouvement social. Ces approches sociologiques nous éclairent sur l'objet général de la recherche: les organisations, leurs caractéristiques et leurs fonctionnements.

A la suite de certains chercheurs ${ }^{11}$, nous constatons que les théories de la communication peuvent compléter de manière pertinente l'analyse sociologique des organisations et définir ainsi le cadre général de la communication organisationnelle.

\section{L'approche communicationnelle}

Comme nous avons pu le voir précédemment, les acteurs d'une mobilisation interagissent, se structurent avec plus ou moins d'efficacité, tentent de conduire leur projet jusqu'à une forme organisationnelle. Mais, comme le précise L. Mathieu,

«Même une fois accompli avec succès, ce travail de ralliement autour d'une cause ne doit pas pour autant être considéré comme allant de soi. Même devant une mobilisation qui a pris, à laquelle se sont ralliés des effectifs parfois considérables de militants, le chercheur se doit de s'interroger sur la consistance (...) du collectif ainsi créé. C'est précisément ce que négligent nombre de définitions qui tendent à considérer la dimension collective des mobilisations comme un donné,

\footnotetext{
${ }^{9}$ Mintzberg H., Le Management. Voyage au centre des organisations, Paris/Montréal, Editions d'Organisation/Agence d'Arc, 1990, pp. 408-435.

${ }^{10}$ Meister A., La participation dans les associations, Paris, Les éditions ouvrières, 1974.

${ }^{11}$ Voir notamment les travaux de N. Giroux, P. de Saint-Georges et C. Duterme.
} 
Dossier : Migrations conceptuelles. D'où viennent les concepts de la communication organisationnelle?

plutôt que d'en pointer les modalités pratiques de constitution, de consolidation et éventuellement de délitement ${ }^{12}$.

C'est bien dans cette perspective que nous nous situons: les organisations de mouvement social que nous souhaitons étudier ne seront pas considérées comme des données au sein desquelles il est possible d'analyser les phénomènes ou politiques de communication. Notre objectif est de traiter du rôle organisant de la communication dans les phases de construction et de consolidation de l'organisation.

Cette partie de l'article précisera la conception de la communication et de la communication organisationnelle- que nous privilégions dans le cadre de notre recherche.

\section{Une conception de la communication}

Les approches sociologiques nous conduisent à considérer l'organisation comme une forme sociale, une configuration, un niveau de régulation de l'action collective. L'acteur collectif d'une mobilisation est amené à cadrer son action, évoluant le long d'un continuum dans le degré de son organisation.

Selon le courant de la pragmatique ${ }^{13}$ de la communication, tout comportement humain est un acte de communication, en interaction avec d'autres, formant ainsi un ensemble, un système. Il s'agit donc de s'intéresser aux codes de comportements interpersonnels et aux éléments qui régulent la dynamique d'un groupe. Ainsi, la communication est un ensemble de comportements et d'opinions en interaction dans un système donné. Elle intègre différents modes de comportements, les codes culturels, les interactions en contexte.

On constate que cette définition de la communication est très proche de la définition de l'organisation précisée ci-avant. Comme le précisent Pierre de Saint-Georges ${ }^{14}$ et Claude Duterme ${ }^{15}$, ceci nous amène à considérer l'organisation directement comme un système de communication. En effet, le courant pragmatique et systémique de la communication, qui considère la communication comme un système

\footnotetext{
${ }^{12}$ Mathieu L., Op. cit., pp. 18-19.

${ }^{13}$ La pragmatique de la communication est née aux Etats-Unis dans les années 50. Les auteurs principaux de ce courant théorique sont, entre autres, G. Bateson, E. Goffman, P. Watzalwick, H. Beavin, R. Birdwhistell ; ils forment ce que l'on appelle «L'école de Palo Alto ».

${ }^{14}$ de Saint-Georges P., «Culture d'entreprise, communication interne et stratégies de changement », in : Communication et Organisation, n ${ }^{\circ} 4,1993$, p. 75.

${ }_{15}$ Duterme C., La communication interne en entreprise. L'approche de Palo Alto et l'analyse des organisations, Bruxelles, De Boeck, 2002, pp. 61-82.
} 
d'interactions, et la sociologie des organisations, qui considère l'organisation comme un système organisé, se complètent et s'articulent ainsi de manière pertinente.

\section{La communication organisationnelle}

Partant de ces définitions de l'organisation et de la communication -et en synthétisant les apports de P. de Saint-Georges ${ }^{16}$ et Nicole Giroux $^{17}$ - il nous faut à présent préciser la conception de la communication organisationnelle qu'elles nous amènent à privilégier : celle de la communication processuelle ou organisante. L'organisation y est directement pensée en tant que système de communication. La communication est constitutive et constituante de l'organisation qui, elle-même, est considérée comme un système de lien social, de personnes en interactions, comme un processus organisant. Cette approche place comme enjeu central le lien social : les personnes sont en interactions dans un collectif, elles créent et appliquent les règles ensemble. Les interactions et les actes de langage sont importants à étudier dans le cadre de cette approche.

L'approche de la communication processuelle ou organisante nous amène à réfléchir à la dimension identitaire des organisations. En effet, les interactions, les engagements et les négociations au sein du collectif -qui sont constituants de l'organisation vue comme système de communication- sont des objets d'analyse extrêmement riche pour la communication organisationnelle et nous révèlent les processus constitutifs de l'identité même de l'organisation. Cette construction identitaire se décèle à la fois dans les interactions et les modes de structuration des organisations (lorsque le projet organisationnel prend forme en interne) et dans les actions communicationnelles portées vers l'extérieur et destinées à façonner l'image de l'organisation.

A ce stade, il est intéressant d'analyser la communication, considérée comme ciment du corps social qui se rassemble autour de l'idée fédératrice, à travers les formes émergentes d'échanges et les influences qui traversent l'organisation que celle-ci incorpore dans ses choix (techniques, stratégiques et humains). L'analyse peut donc porter sur les opérations et dispositifs de traitement d'informations

\footnotetext{
16 de Saint-Georges P., Cours de Communication et organisation, COMU/ESPO/UCL.

17 Giroux N., «La communication interne: une définition en évolution », in Communication et Organisation, n5, 1994, pp. 17-46 et Giroux N., «Vers une approche processuelle de la communication du changement en organisation », exposé au Colloque «L'organisation Média », Lyon, Université Jean-Moulin Lyon 3, 20 novembre 2004.
} 
Dossier : Migrations conceptuelles. D'où viennent les concepts de la communication organisationnelle?

(par exemple : la charte de l'organisation, qui rend compte de son activité de médiatisation), et sur les modalités d' "être ensemble » (menant à la collaboration, la coordination et l'adhésion). Certains documents communicationnels peuvent également être analysés, car ils permettent de diffuser des idées, mais également parce qu'ils laissent des traces, permettant à l'organisation de s'auto-évaluer, d'évoluer, de continuer dans l'une ou l'autre direction.

\section{Au croisement : une approche communicationnelle des organisations de mouvement social}

On le constate, approches sociologiques et communicationnelles se complètent, s'articulent pour éclairer l'analyse des organisations. La sociologie nous apporte une connaissance approfondie des organisations de mouvement social; ce faisant, des concepts et processus communicationnels sont mis en exergue, tels que l'environnement du mouvement social, les flux d'informations, les réseaux, les mécanismes de coordination autour du projet, les processus interactionnels... La communication est un phénomène central de l'organisation, la communication est organisante. Ceci fonde une approche -ou une perspective- communicationnelle des organisations, qui se base sur les travaux sociologiques.

Sur base de ce cadre théorique, nous avons construit le cadre d'analyse de notre recherche, composé d'une analyse sémiopragmatique des discours d'organisations, enrichie des apports d'un type d'analyse souvent utilisée dans les études des mouvements sociaux, l'«analyse des cadres». En effet, dans la vision de la communication processuelle ou organisante, les récits ou discours formalisent et accompagnent le processus d'organisation sociale. Ils sont le lieu où se construisent les acteurs et l'organisation elle-même, ils proposent des signes de reconnaissance et d'appartenance. Ainsi se profile un cadre d'analyse des organisations de mouvement social qui pourra faire l'objet d'une étude empirique.

\section{L'analyse sémio-pragmatique des discours d'organisations}

La méthode proposée vise à analyser les discours des organisations altermondialistes (chartes, projets, appels à mobilisations, prises de parole publiques, tracts...). Cette démarche n'appréhende ni l'organisation textuelle en elle-même, ni la situation de communication, mais s'efforce de les associer. Le texte est analysé comme activité énonciative rapportée à un genre de discours : le lieu social dont il émerge, le canal par lequel il passe, le type de diffusion qu'il implique... ne sont pas dissociables de la façon dont le texte 
s'organise ${ }^{18}$. Cette analyse des discours organisationnels nous permettra de déceler ce que Nicole d'Almeida appelle les récits de la maisonnée et les récits de l'engagement ${ }^{19}$.

L'analyse des discours d'organisations altermondialistes nous amènera à utiliser l'analyse sémio-pragmatique. Selon Gino Gramaccia $^{20}$, le lien de coopération unissant des individus engagés dans des dispositifs organisationnels est créé par certaines pratiques langagières relevant de la pragmatique. La théorie des actes de langage d'Austin s'ancre ici dans le domaine organisationnel. Selon James R. Taylor ${ }^{21}$, rien ne s'oppose à l'idée selon laquelle l'organisation est constituée de liens de réciprocité, de coopération, dont la consistance, la solidité, mais aussi l'impact ou la dimension stratégique sont de nature pragmatique. L'auteur met donc en avant le caractère pragmatique des liens organisationnels dont le «tissage" repose sur des propriétés illocutionnaires. Les acteurs structurent leurs relations et engagent des actions à partir de procédés langagiers « qui font des choses avec des mots ». Ainsi se dessine le projet d'une approche de la communication organisationnelle dans laquelle l'interaction pragmatique occupe une place centrale.

L'analyse sémio-pragmatique des discours organisationnels nous semble une piste intéressante pour l'étude de la communication des organisations de mouvement social. Afin de préciser le type d'analyse qui pourrait être mené, nous souhaitons la croiser avec l'« analyse des cadres ».

\section{L'analyse des cadres}

L' " analyse des cadres » de l'expérience contestataire est l'application au domaine des mouvements sociaux d'un modèle

\footnotetext{
${ }^{18}$ Nous nous attacherons donc à comprendre le fonctionnement organisationnel et le contexte de production des discours des organisations altermondialistes choisies, grâce à des observations, des entretiens semi-dirigés auprès des membres et des recherches documentaires.

${ }^{19}$ Dans son approche narratologique des discours tenus dans et par les organisations, $\mathrm{N}$. d'Almeida distingue un premier type de récits mettant en scène des valeurs internes, dressant les contours d'une identité particulière. Il s'agit de délimiter un territoire de buts et de relations et de raffermir les liens à l'intérieur d'un groupe. Le deuxième type de récits correspond à un mouvement d'extension et d'ouverture où une adhésion à des valeurs universelles est proposée à l'opinion, où la mission est exposée. Voir à ce propos d'Almeida N., Les promesses de la communication, Paris, PUF, 2001.

${ }^{20}$ Gramaccia G., Les actes de langage dans les organisations, Paris, L’Harmattan, 2001.

${ }^{21}$ Cité dans Gramaccia G., Op. cit.
} 
Dossier : Migrations conceptuelles. D'où viennent les concepts de la communication organisationnelle?

théorique initialement construit pour rendre compte d'activités d'autres types. Les cadres de l'expérience, selon Erving Goffman ${ }^{22}$ sont les principes à partir desquels les individus comprennent et donnent sens aux différentes situations qu'ils rencontrent dans leur vie quotidienne. La prise en compte des composantes cognitives et discursives de l'action collective s'est ainsi traduite par le recours à la notion de «cadres ». Le point d'ancrage de l'«analyse des cadres» est bien celui-là : les mouvements d'action collective doivent être appréhendés comme des «producteurs de significations». Ils sélectionnent -cadrent- certaines formes de vie collective et publique plutôt que d'autres.

Les organisations de mouvement social procèdent, à travers leurs discours, à des opérations de cadrage ayant trois fonctions: le diagnostic (opérations visant à trouver des causes, à attribuer des responsabilités, à blâmer des coupables, à identifier des victimes dans une situation problématique), le pronostic (formulation d'une solution possible et son articulation sur la situation problématique, ainsi que les stratégies d'action qui vont en découler), et le cadrage motivationnel (production des raisons de l'engagement dans l'action et sélection de vocabulaire de motifs appropriés).

Tout au long de son parcours, une organisation de mouvement social se forme, se donne un programme, propose des slogans et des projets rassemblant des membres qui se coordonnent et justifient leurs actions, recrute des militants... Selon David $\mathrm{Snow}^{23}$, l'alignement des cadres est une activité qui permet à l'organisation de mener ces différentes étapes. Il s'agit d'une activité consciente des « entrepreneurs » de l'organisation ou des militants locaux, visant la constitution de soutien par une mise en résonance entre des schèmes interprétatifs produits par un mouvement et ceux présents ou latents dans des publics cibles. En fonction des publics auxquels s'adresse l'organisation, et selon que les locuteurs tentent de s'accorder aux perceptions des individus qu'ils cherchent à recruter ou de modifier ces perceptions afin de les convaincre de participer à l'action collective, l'alignement sera différent (connexion, amplification, extension ou transformation des cadres).

\footnotetext{
${ }^{22}$ Goffman E., Les cadres de l'expérience, Paris, Minuit, 1974.

${ }^{23}$ Snow D., et al., « Frame Alignement Processes, Micromobilization, and Movement Participation », in : American Sociological Review, vol. 51, cité dans Mathieu, L., Op. cit.
} 
Notre recherche visera donc à travailler sur l'expressivité, c'est-à-dire la traduction des activités du groupe par des actions collectives dans l'espace public. Il s'agit d'étudier le travail symbolique de l'organisation de mouvement social. Nous proposons d'effectuer cette étude de l'expressivité des organisations de mouvement social à travers l'analyse sémio-pragmatique du discours, comportant toute une série d'opérations de cadrage. L'analyse des cadres vient préciser l'analyse des discours : les cadres peuvent avoir une efficacité venant des logiques discursives, des mises en récit qu'il est important de prendre en compte.

Notre objectif est bien d'étudier les évolutions discursives des organisations choisies et ainsi déceler les traces des évolutions organisationnelles, des phases de passage au débat public du projet. Bien que limitée vu le caractère récent de la constitution des organisations étudiées, l'analyse des discours nous permettra de déceler les éventuels changements discursifs au fil des années d'existence des collectifs.

A nouveau -mais ici concernant les méthodologies d'analyse de contenu- approches sociologiques et communicationnelles s'articulent de manière pertinente.

\section{Conclusion}

Cet article a retracé le parcours qui nous a menée à la constitution d'un cadre théorique pour notre recherche: l'approche communicationnelle des organisations de mouvement social.

$\mathrm{Ce}$ cadre théorique articule approches sociologiques et communicationnelles: la sociologie des organisations, des mouvements sociaux et des associations nous apporte une connaissance approfondie des organisations de mouvement social ; les théories de la communication complètent ce cadre pour définir la communication organisationnelle. En retraçant rapidement les éclairages sociologiques, on constate que les phénomènes et processus communicationnels y sont centraux; la communication est organisante. Ainsi se dessine une approche communicationnelle des organisations croisant les apports de la sociologie des organisations et des sciences de la communication.

Cette approche nous amène à nous intéresser particulièrement aux discours des organisations et à proposer des pistes concrètes d'analyse. Sur le plan des méthodologies de recherche, les approches utilisées en sociologie des mouvements sociaux et en information et communication s'articulent également. L'analyse des cadres et 
Dossier : Migrations conceptuelles. D'où viennent les concepts de la communication organisationnelle?

l'analyse sémio-pragmatique des discours nous permettront de déceler en quoi ces « textes » sont des éléments structurants des organisations altermondialistes. Lors de la phase d'interprétation des résultats, un retour nécessaire se fera sur les concepts mis en exergue par l'approche communicationnelle des organisations de mouvement social.

\section{Bibliographie}

Brulois V., "Communication et organisation : état des recherches en sciences de l'information et de la communication ", in Education permanente, $\mathrm{n}^{\circ} 16,2006$, p. 29-41.

d'Almeida N., Les promesses de la communication, Paris, PUF, 2001.

Della Porta D., Diani M., Social Movements. An Introduction, Oxford, Blackwell, 1999.

de Saint-Georges P., «Culture d'entreprise, communication interne et stratégies de changement", in Communication et Organisation, $\mathrm{n}^{\circ} 4,1993$, p. 71-93.

Duterme C., La communication interne en entreprise. L'approche de Palo Alto et l'analyse des organisations, Bruxelles, De Boeck, 2002.

Friedberg E., "Les quatre dimensions de l'action organisée », in Revue française de sociologie, vol. 33, 1992, 4, p. 531-557.

Giroux N., « La communication interne: une définition en évolution », in : Communication et Organisation, n5, 1994, p. 17-46.

Giroux N., "Vers une approche processuelle de la communication du changement en organisation », exposé au Colloque "L'organisation Média », Lyon, Université Jean-Moulin Lyon 3, 20 novembre 2004.

Goffman E., Les cadres de l'expérience, Paris, Minuit, 1974.

Gramaccia G., Les actes de langage dans les organisations, Paris, L'Harmattan, 2001.

Laville J.-L., Sainsaulieu R., Sociologie de l'association, Paris, Desclée de Brouwer, 1997.

Mathieu L., Comment lutter? Sociologie et mouvements sociaux, Paris, Les éditions Textuel, 2004.

Meister A., La participation dans les associations, Paris, Les éditions ouvrières, 1974. 
La construction d'une approche communicationnelle...

Mintzberg H., Le Management. Voyage au centre des organisations, Paris/Montréal, Editions d'Organisation/Agence d'Arc, 1990.

Neveu E., Sociologie des mouvements sociaux, Paris, La Découverte, Repères 207, 2000.

Oberschall A., Social conflict and social movements, Englewood Cliffs, Prentice Hall, 1973.

Snow D., et al., «Frame Alignement Processes, Micromobilization, and Movement Participation », in: American Sociological Review, vol. 51.

Walgrave S., "Nieuwe sociale bewegingen: een type van sociale bewegingen », in : Tijdschrift voor Sociologie, 13, 1, p. 71-104. 